Research Paper

\title{
The role of postoperative radiotherapy (PORT) in combined small cell lung cancer (C-SCLC)
}

\author{
Yu Men ${ }^{1, *}$, Yang Luo ${ }^{2, *}$, Yirui Zhai ${ }^{1}$, Jun Liang ${ }^{1}$, Qinfu Feng ${ }^{1}$, Dongfu Chen ${ }^{1}$, Zefen \\ $\mathrm{XiaO}^{1}$, Zongmei Zhou ${ }^{1}$, Zhouguang $\mathrm{Huil}^{3}$ and Luhua Wang ${ }^{1}$ \\ ${ }^{1}$ Department of Radiation Oncology, National Cancer Center/Cancer Hospital, Chinese Academy of Medical Sciences and \\ Peking Union Medical College, Beijing 100021, China \\ ${ }^{2}$ Department of Medical Oncology, National Cancer Center/Cancer Hospital, Chinese Academy of Medical Sciences and Peking \\ Union Medical College, Beijing 100021, China \\ ${ }^{3}$ Department of VIP Medical Services \& Department of Radiation Oncology, National Cancer Center/Cancer Hospital, Chinese \\ Academy of Medical Sciences and Peking Union Medical College, Beijing 100021, China \\ *These authors contributed equally to this work \\ Correspondence to: Zhouguang Hui, email: drhuizg@163.com \\ Luhua Wang, email: wlhwq@yahoo.com
}

Keywords: carcinoma, combined small cell lung, surgery, radiotherapy, survival

Received: December 07, $2016 \quad$ Accepted: March 22, $2017 \quad$ Published: April 06, 2017

Copyright: Men et al. This is an open-access article distributed under the terms of the Creative Commons Attribution License 3.0 (CC BY 3.0), which permits unrestricted use, distribution, and reproduction in any medium, provided the original author and source are credited.

\section{ABSTRACT}

Purpose: To explore the value of radiotherapy in C-SCLC patients, especially in those receiving a radical resection.

Results: The differences of survivals between the postoperative radiotherapy (PORT) and non-PORT groups were not statistically significant. But analyzing the benefits in subgroups, PORT significantly improved OS $(p=0.015)$, DFS $(p=0.026)$, LRFS $(p=0.008)$ and DMFS $(p=0.030)$ in stage III patients. For the patients with N2 stage, all survivals of the PORT group were also statistically significantly higher than non-PORT group $(p=0.018,0.032,0.008,0.042)$. Patients with more than $10 \%$ of metastatic lymph nodes could get a significant benefit survivals by receiving PORT $(p=0.033,0.030,0.025,0.031)$. Having a systematic dissection of more than 17 lymph nodes was a subset which could get better OS and LRFS by receiving PORT $(p=0.045,0.048)$.

Methods: Between Jan. 2004 to Dec. 2012, fifty-five patients diagnosed as C-SCLC after complete surgical resection in our center were retrospectively analyzed. The overall survival (OS), disease free survival (DFS), loco-regional recurrence free survival (LRFS), and distant metastasis free survival (DMFS) were calculated by Kaplan-Meier method.

Conclusions: PORT can significantly improve the survival of C-SCLC patients with resected pathological pN2 stage. For the patients with a large percent of metastatic lymph nodes, PORT can also improve survivals.

\section{INTRODUCTION}

Combined small cell lung cancer (C-SCLC) is defined as small cell lung cancer (SCLC) combined with an additional component that consists of any of the histological types of non-small cell lung cancer (NSCLC). $\mathrm{C}$-SCLC is quite an uncommon cancer possessing about $1-3 \%$ of all SCLCs [1-5].

The diagnosis of C-SCLC mainly depends on complete examination of pathological specimen after surgery. With the development of screening and surgical techniques, more and more patients with early lung cancer are diagnosed and have the opportunity to receive surgery and complete pathological examination as well, which led to the increase of diagnosed C-SCLC. Therefore, it is necessary to explore the value of postoperative radiotherapy (PORT).

PORT improves the treatment results in patients with $\mathrm{pN}+\mathrm{SCLC}$, as well as those with pIIIA-N2 NSCLC. But PORT for C-SCLC is suboptimized although the treatment 
of C-SCLC mainly refers to the guideline of SCLC. Furthermore, due to the low incidence and lack of attention, there is no study focusing on PORT for C-SCLC yet. Our single institutional study aimed to elucidate the effectiveness of PORT on survival of C-SCLC patients and to identify the subgroups which may most likely benefit from PORT.

\section{RESULTS}

\section{Patient characteristics}

Totally 55 consecutive patients were enrolled. Characteristics of the patients are presented in (Table 1). The median age of the whole group was 58 years (range, $35-76$ years). Thirty patients $(54.5 \%)$ had the component of squamous cell carcinoma (SCC). The $\mathrm{T}$ stage was $\mathrm{T}_{1-2}$ in 34 patients $(61.8 \%)$ and $\mathrm{T}_{3-4}$ in 21 patients $(38.2 \%)$. The $\mathrm{N}$ stage was $\mathrm{N}_{0}$ in 23 patients $(41.8 \%), \mathrm{N}_{1}$ in 14 patients $(25.5 \%)$ and $\mathrm{N}_{2}$ in 18 patients $(32.7 \%)$, respectively. In addition, stage III counted the most by $47.3 \%$. Regardless of the sequence, most patients received chemotherapy $(76.4 \%)$. Of all 55 patients, 14 $(25.5 \%)$ received PORT. Twelve out of the 14 patients had detailed records of radiation and all received intensity modulated radiation therapy. The median total radiation dose was 60 Gy (range, 50Gy-66Gy). Seven
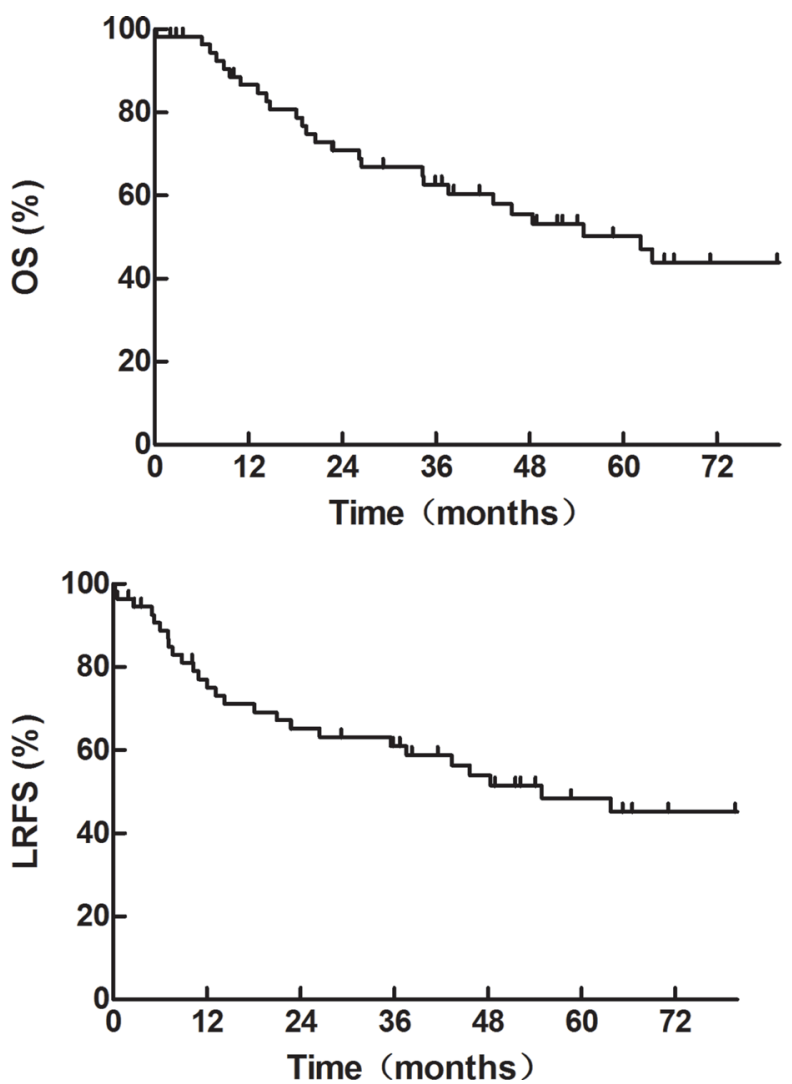

patients received a total dose $\geq 60$ Gy and 5 patients less than 60Gy. The clinical characteristics were comparable between the PORT group and non-PORT group, except that there were more $\mathrm{N}_{0-1}$ patients and more patients receiving chemotherapy and less patients receiving $\mathrm{PCI}$ in the non-PORT group (as shown in Table 1).

\section{Survivals in the whole group}

The median follow-up was 56.4 months (range, 2.0-127.2 months). The median OS, DFS, LRFS and DMFS were 62.2 months, 43.4 months, 54.9 months and 43.4 months, respectively. As shown in (Figure 1), the 1-, 3 -, and 5-year OS rates were $86.6 \%, 62.6 \%$ and $50.2 \%$, respectively, and the corresponding DFS rates were $70.1 \%, 54.6 \%$, and $42.8 \%$. The 1-, 3-, and 5-year LRFS rates were $77.1 \%, 61.1 \%$ and $48.4 \%$, respectively, and the corresponding DMFS rates were $75.7 \%, 56.6 \%$, and $42.5 \%$.

\section{Effect of radiotherapy on survivals}

For patients in the PORT and non-PORT groups, the median OS were 54.9 months and 62.2 months, respectively $\left(\chi^{2}=0.267, p=0.605\right)$ (Figure $\left.2 \mathrm{~A}\right)$. The $1-$, 3 - and 5-year OS rates were $92.9 \%, 69.6 \%$ and $48.8 \%$ in the PORT group, and $84.4 \%, 60.1 \%$ and $50.4 \%$ in the
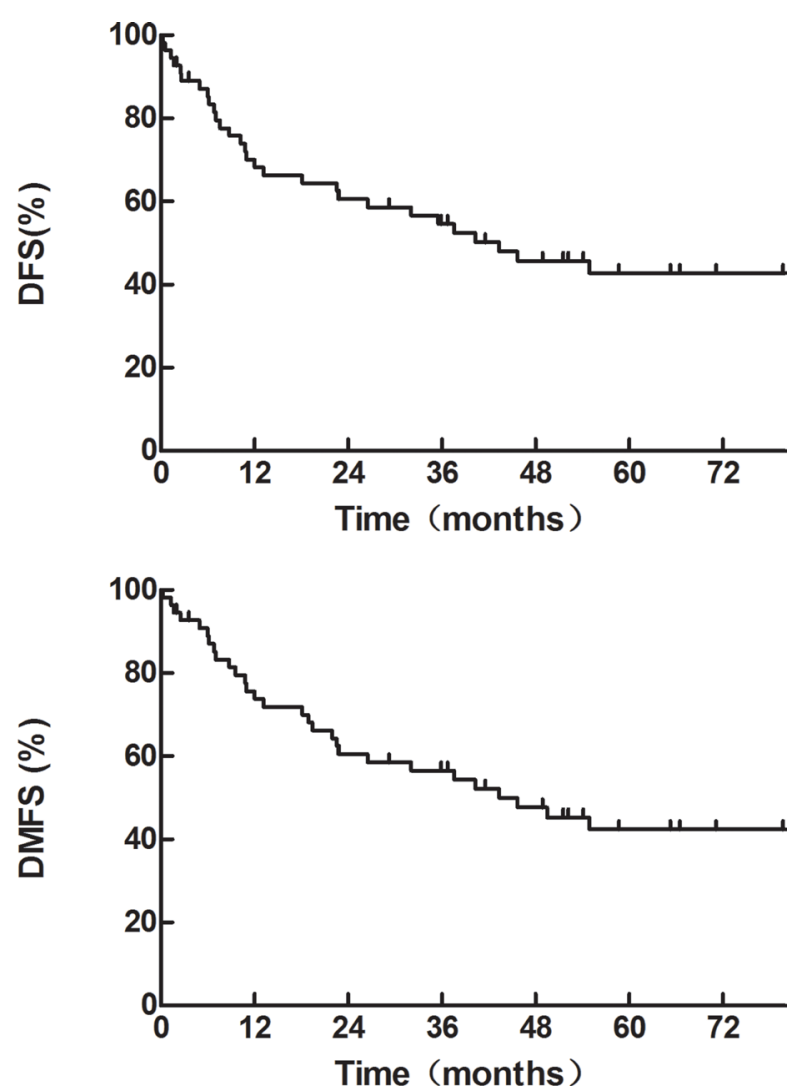

Figure 1: OS, DFS, LRFS and DMFS of the whole group of 55 patients. 
Table 1: Patient characteristics $(N=55)$

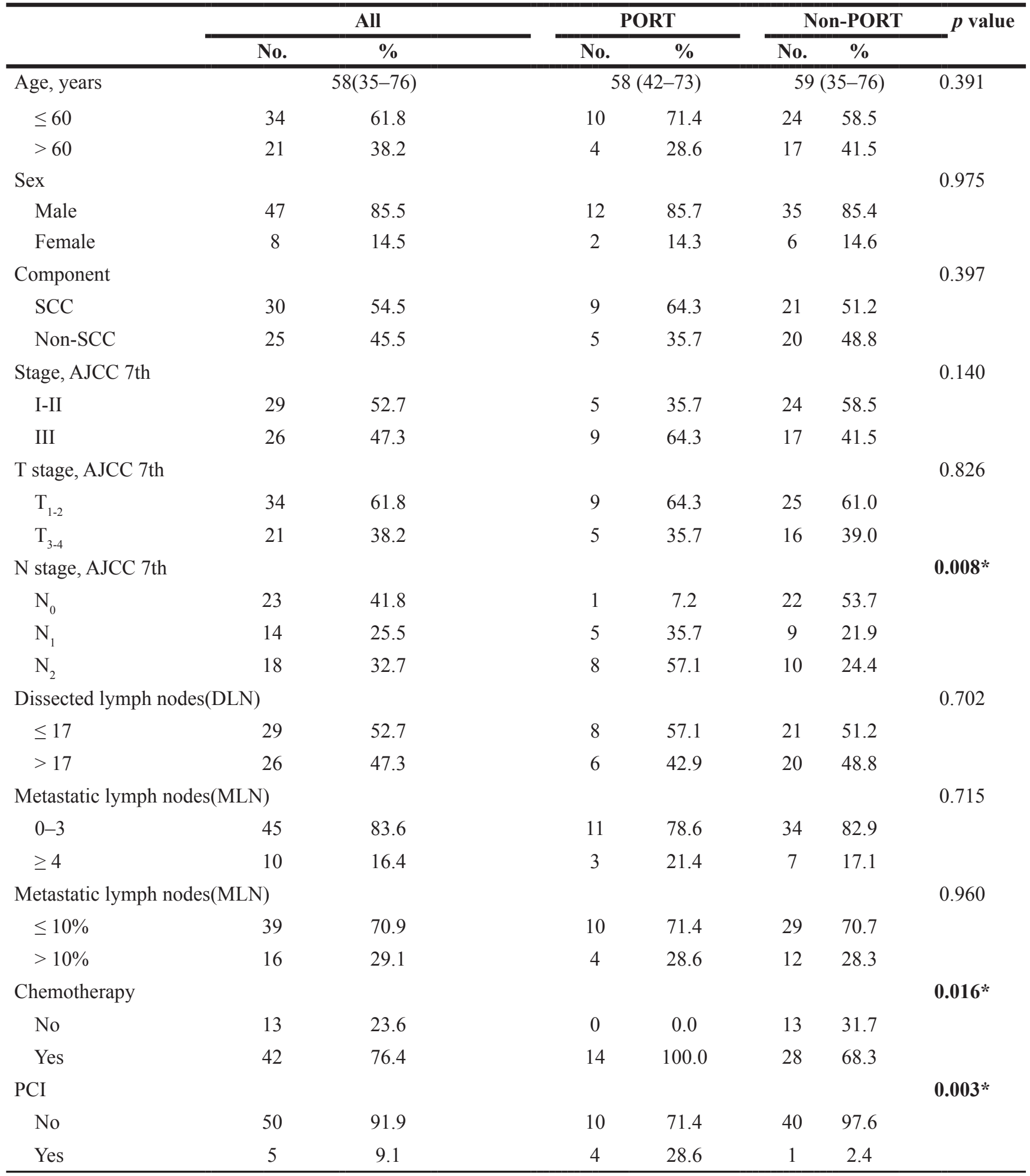

non-PORT group, respectively. The 1-, 3- and 5-year DFS rates were $85.7 \%, 64.3 \%$ and $45.0 \%$ in the PORT group, and $64.5 \%, 51.1 \%$ and $41.8 \%$ in the non-PORT group, respectively $\left(\chi^{2}=0.532, p=0.466\right)$ (Figure 2B). The 1-, 3-, and 5-year LRFS rates were 92.9\%, 69.6\%, and $48.8 \%$ in the PORT group and $71.4 \%, 58.0 \%$, and $48.1 \%$ in the non-PORT group, respectively $\left(\chi^{2}=0.558\right.$, $p=0.455)$ (Figure 2C). The 1-, 3- and 5-year DMFS rates were $85.7 \%, 64.3 \%$ and $45.0 \%$ in the PORT group, and $72.0 \%, 53.8 \%$ and $41.4 \%$ in the non-PORT 


\begin{tabular}{|c|c|c|c|c|c|c|c|c|c|c|c|c|}
\hline \multirow{2}{*}{ Factor } & \multicolumn{3}{|c|}{$5 y-O S(\%)$} & \multicolumn{3}{|c|}{ 5y-DFS (\%) } & \multicolumn{3}{|c|}{ 5y-LRFS (\%) } & \multicolumn{3}{|c|}{ 5y-DMFS (\%) } \\
\hline & PORT & $\begin{array}{l}\text { Non- } \\
\text { PORT }\end{array}$ & $\begin{array}{c}p \\
\text { value }\end{array}$ & PORT & $\begin{array}{l}\text { Non- } \\
\text { PORT }\end{array}$ & $\begin{array}{c}p \\
\text { value }\end{array}$ & PORT & $\begin{array}{l}\text { Non- } \\
\text { PORT }\end{array}$ & $p$ value & PORT & $\begin{array}{l}\text { Non- } \\
\text { PORT }\end{array}$ & $p$ value \\
\hline \multicolumn{13}{|c|}{ Stage, AJCC 7th } \\
\hline I-II & 30.0 & 75.2 & 0.165 & 30.0 & 59.5 & 0.489 & 30.0 & 70.4 & 0.232 & 30.0 & 58.8 & 0.423 \\
\hline III & 61.0 & 13.4 & $0.015^{*}$ & 53.3 & 13.8 & $0.026^{*}$ & 61.0 & 13.7 & $0.008^{*}$ & 53.3 & 13.6 & $0.030^{*}$ \\
\hline \multicolumn{13}{|c|}{ T stage, AJCC 7th } \\
\hline $\mathrm{T}_{1-2}$ & 56.3 & 61.7 & 0.922 & 50.0 & 59.4 & 0.993 & 56.3 & 62.3 & 0.842 & 18.6 & 59.4 & 0.939 \\
\hline $\mathrm{T}_{3-4}$ & 30.0 & 31.3 & 0.498 & 30.0 & 14.8 & 0.318 & 30.0 & 23.7 & 0.362 & 30.0 & 14.7 & 0.405 \\
\hline \multicolumn{13}{|c|}{$\mathrm{N}$ stage, AJCC 7th } \\
\hline $\mathrm{N}_{0}$ & 0.0 & 83.8 & 0.115 & 0.0 & 64.9 & 0.457 & 0.0 & 78.6 & 0.201 & 0.0 & 64.2 & 0.468 \\
\hline $\mathrm{N}_{+}$ & 57.5 & 11.8 & $0.012^{*}$ & 52.7 & 12.1 & $0.006^{*}$ & 57.5 & 12.0 & $0.003^{*}$ & 52.7 & 11.9 & $0.010^{*}$ \\
\hline $\mathrm{N}_{1}$ & 40.0 & 12.7 & 0.313 & 40.0 & 13.0 & 0.135 & 40.0 & 12.7 & 0.183 & 40.0 & 13.0 & 0.188 \\
\hline $\mathrm{N}_{2}$ & 72.9 & 11.1 & $0.018^{*}$ & 62.5 & 11.4 & $0.032 *$ & 72.9 & 11.4 & $0.008^{*}$ & 62.5 & 11.1 & $0.042^{*}$ \\
\hline \multicolumn{13}{|l|}{ DLN } \\
\hline$\leq 17$ & 16.7 & 49.9 & 0.248 & 16.7 & 43.3 & 0.599 & 16.7 & 50.5 & 0.410 & 16.7 & 43.3 & 0.519 \\
\hline$>17$ & 100 & 51.2 & $0.045^{*}$ & 83.3 & 40.2 & 0.109 & 100 & 46.0 & $0.048^{*}$ & 83.3 & 39.3 & 0.121 \\
\hline \multicolumn{13}{|l|}{ MLN } \\
\hline $0-3$ & 38.9 & 60.0 & 0.758 & 35.4 & 49.3 & 0.990 & 38.9 & 56.9 & 0.933 & 35.4 & 48.9 & 0.896 \\
\hline$\geq 4$ & 66.7 & 0.0 & 0.155 & 66.7 & 0.0 & 0.153 & 66.7 & 0.0 & 0.096 & 66.7 & 0.0 & 0.161 \\
\hline$\leq 10 \%$ & 37.0 & 69.4 & 0.332 & 33.3 & 55.9 & 0.549 & 37.0 & 66.0 & 0.474 & 50.0 & 55.5 & 0.454 \\
\hline$>10 \%$ & 75.0 & 10.0 & $0.033^{*}$ & 75.0 & 0.0 & $0.030^{*}$ & 75.0 & 0.0 & $0.025^{*}$ & 75.0 & 0.0 & $0.031^{*}$ \\
\hline \multicolumn{13}{|l|}{ Component } \\
\hline $\mathrm{SCC}$ & 54.7 & 56.3 & 0.402 & 48.6 & 44.7 & 0.480 & 54.7 & 50.8 & 0.311 & 48.6 & 43.8 & 0.526 \\
\hline Non-SCC & 40.0 & 45.0 & 0.704 & 40.0 & 39.4 & 0.924 & 40.0 & 45.3 & 0.913 & 40.0 & 39.4 & 0.990 \\
\hline
\end{tabular}

group, respectively $\left(\chi^{2}=0.367, p=0.544\right)$ (Figure 2D). Though the OS, DFS, LRFS and DMFS all trended to be better in the PORT group than in non-PORT group, the differences were not statistically significant. On multivariate analysis, PORT was not significant positive prognostic factors for $\mathrm{OS}(\mathrm{HR}=0.516,95 \% \mathrm{CI}$ $0.194-1.371, p=0.185)$.

\section{Subgroup analyses}

Results of the subgroup analyses are shown in (Table 2). PORT significantly improved the survivals of patients with stage III or $\mathrm{N}_{2}$ disease, as well as those with more than $10 \%$ of metastatic lymph nodes. For the patients with stage III disease, the 1-, 3- and 5-year OS 
rates were $88.9 \%, 76.2 \%$ and $61.0 \%$ in the PORT group, which were statistically significantly higher than the corresponding rates of $67.2 \%, 20.2 \%$ and $13.4 \%$ in the non-PORT group $(p=0.015)$. The DFS, LRFS and DMFS in the PORT group were also significantly improved comparing with the non-PORT group $(p=0.026,0.008$ and 0.030 , respectively). For patients with $\mathrm{N}_{+}$disease, the OS, DFS, LRFS and DMFS in the PORT group were significantly higher than those in the non-PORT group ( $p$ $=0.012,0.006,0.003$ and 0.010 , respectively). However, when analyzed $\mathrm{N}_{1}$ and $\mathrm{N}_{2}$ separately, the significant difference only existed in $\mathrm{N} 2$ disease $(p=0.018,0.032$, 0.008 and 0.042 , respectively) while the difference was marginally seen in $\mathrm{N} 1$ disease. Accordingly, patients with more than $10 \%$ of metastatic lymph nodes also had better survivals in the PORT group ( $p=0.033,0.030,0.025$ and 0.031 , respectively). Moreover, Patients having more than 17 DLNs had a significantly better OS $(p=0.045)$ and LRFS $(p=0.048)$, but not better DFS $(p=0.109)$ or DMFS $(p=0.121)$ in the PORT group than the non-PORT group. PORT also markedly increased the survival rates in patients with $\mathrm{T}_{3-4}$ tumors or with $\geq 4$ positive lymph nodes, but the difference was not statistically significant which may due to the small samples. On the contrary, PORT seemed to deteriorate the survivals in patients
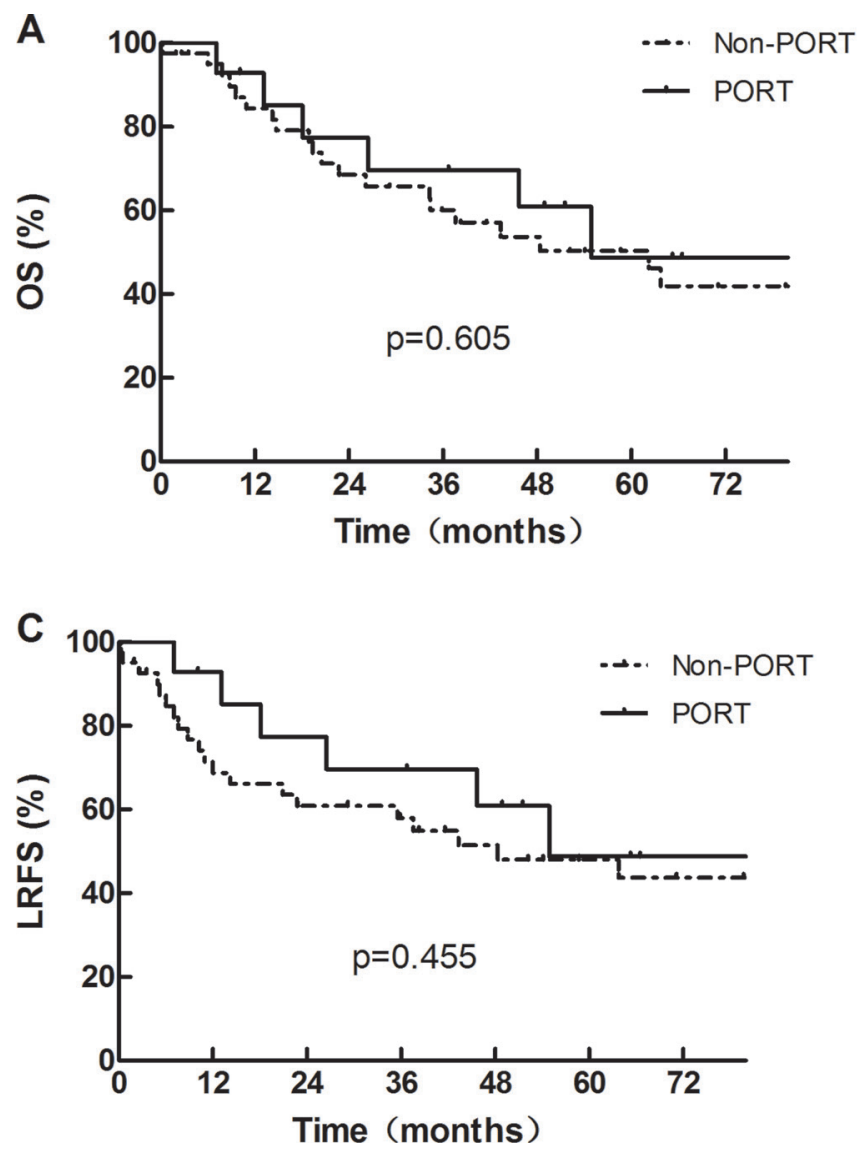

with $\mathrm{T}_{1-2}$ or I-II disease though the difference was not statistically significant. Whether or not having SCC was not a significant factor for PORT. As for the patients with smoking history, PORT did not influence the OS ( $p=$ $0.843)$, DFS $(p=0.901)$, LRFS $(p=0.945)$ and DMFS $p$ $=0.987)$. In the patients who received chemotherapy, the PORT group had similar survivals to the non-PORT group (OS, $p=0.702$; DFS, $p=0.681$; LRFS, $p=0.508$; and DMFS, $p=0.777)$.

\section{DISCUSSION}

C-SCLC contains the component of both SCLC and NSCLC. However, there are different applications of PORT between SCLC and NSCLC. PORT improves the treatment results in patients with $\mathrm{pN}+\mathrm{SCLC}$, but those with pIIIA-N2 in NSCLC. Therefore, it's a question that whether C-SCLC should follow the guideline of SCLC or NSCLC or having its own when confronting the selection of PORT, especially in current circumstances that more and more patients were diagnosed as C-SCLC after surgery.

The rationale behind PORT is to kill malignant cells remaining in tumor bed, at the resection margins, or in the adjacent lymph nodes after surgery and so to reduce
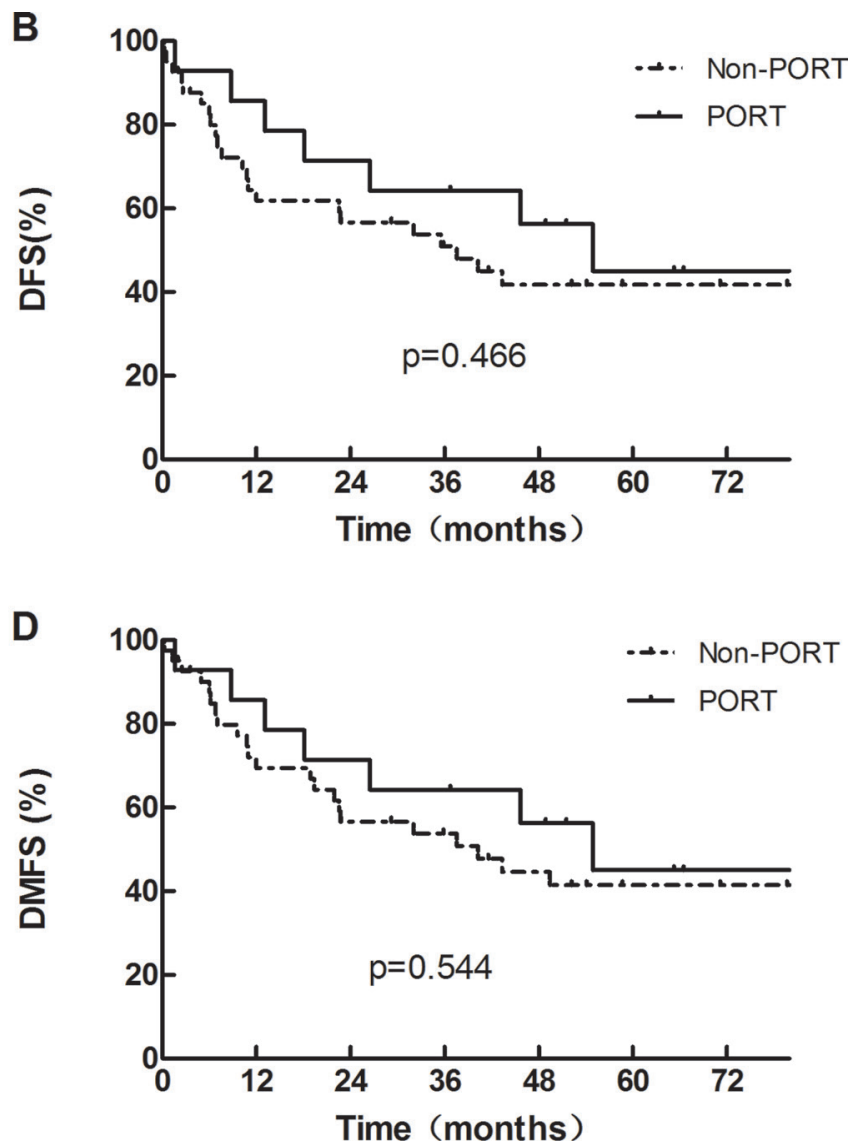

Figure 2: OS, DFS, LRFS and DMFS of the whole group of 55 patients, divided according to whether or not receiving PORT. 
local and regional recurrences and improve survival. At present, the selection of PORT in C-SCLC mainly depends on the experience of doctors, lacking of clinical evidences. In both SCLC and NSCLC, PORT has certain indications. If giving PORT indiscriminately, there are clear evidences showing no benefit, even detrimental effects for patients with completely resected lung cancer $[6,7]$. The metaanalysis published in 1998, which contained 9 randomized controlled trials and 2128 NSCLC patients, concluded that PORT was detrimental (HR 1.21, $p=0.001$ ): twenty-one percent increase in the relative risk of death and 2-year survival rates of $48 \%$ for PORT and $55 \%$ for non-PORT group [6]. The updated results were largely unchanged [8]. Liu et al. [7] revealed that, in SCLC, the OS in the whole PORT group comparing with the non-PORT group was not significantly increased $(p=0.26)$. Consistently, our results show that for whole group, the survival rates are not different in the PORT group and non-PORT group. On multivariate analysis, PORT was also not significant prognostic factors. Therefore, recognizing the subgroups that could benefit from PORT is more meaningful.

In the treatment of SCLC, PORT is recommended to the patients with positive lymph nodes through the detection of surgical specimens. Liu et al. [7] revealed that PORT significantly reduced local-regional recurrence (LRR) and improved OS in patients with regional metastasis SCLC. In patients with N1 disease, the median OS were 40 months in the PORT group versus 14 months in the non-PORT group $(p=0.032)$. The corresponding OS in N2 patients were 35 months versus 17 months, respectively $(p=0.040)$. For patients with $\mathrm{N} 1$ disease, the 3 -year LRR rate was $0.0 \%$ in the PORT group versus $14.3 \%$ in the non-PORT group ( $p=$ $0.037)$. The corresponding LRR rate in N2 patients was $4.2 \%$ versus $56.6 \%(p<0.001)$. For NSCLC, the subset analysis of meta-analysis published in 1998 indicated that in patients with stage III or $\mathrm{pN}_{2}$, survival was slightly better with PORT [6]. In 2006, a retrospective analysis of the SEERs database including 7,465 patients also showed an increase in overall survival in $\mathrm{pN}_{2}$ disease (HR $=0.855, p=0.0077)$ [9]. Dai et al. [10] presented that PORT could significantly improve the survival of patients with resected pathological stage IIIA- $\mathrm{N}_{2}$ NSCLC. Recently, Patel et al. [11] conducted a metaanalysis to evaluate the role of PORT based on the use of linear accelerators in $\mathrm{pN}_{2}$ lung cancer. The $\mathrm{OS}$ and LRFS of PORT group had both significantly improved (HR 0.77, $p=0.020$; HR 0.51, $p<0.001$, respectively). In our study, similarly as SCLC and NSCLC, PORT could significantly improve OS, DFS, LRFS and DMFS of C-SCLC patients with stage III or pN2 stage. For these stages of C-SCLC, PORT may be recommended. Nowadays, the treatment of C-SCLC mainly refers to the guideline of SCLC. However, unlike SCLC recommending PORT in N1 disease, C-SCLC with N1 disease could not get benefit from PORT, which may due to the component of NSCLC. In the other hand, PORT didn't influence the survival of patients with component of SCC or with non-SCC. This indicates that, although the component of NSCLC may affect the use of PORT, the specific kind of NSCLC makes no difference.

The survival rates of patients with a large ratio of involved lymph nodes were significantly improved when receiving PORT. It has been reported that the number of metastatic mediastinal lymph nodes was a significant prognostic factor. Local and distant control are decreased with the increase of positive hilar or mediastinal lymph nodes [12-14]. Other than the number of MLN, the ratio of MLN or the lymph node ratio (LNR) has been proposed as a more useful prognostic metric because of its incorporation of both the number of positive nodes and the total number of examined nodes. Damien et al. [15] analyzed the benefit of PORT by nodal stage and LNR. It turned out that a high LNR was associated with a poorer survival in resected node-positive NSCLC. This may be the reason why PORT could improve survival of these patients.

PORT may have an important position in the treatment of patients having more DLN. The frequency of distant metastases is still very high after the resection of primary tumor and cleaning of regional lymph nodes. Even more, the rate of distant metastases is significantly higher than the incidence of local failure. Therefore, a survival benefit of PORT could only be acquired if microscopic metastatic disease is also effectively controlled. The more lymph nodes being excised, the less possibility of remaining disease. Thus the benefits obtained by using PORT can bring out.

As a retrospective analysis, our study has some limitations. First, all of the patients came from our single institution and the number of cases is limited. The results should be interpreted cautiously as selection bias may exist. Second, the median dose of RT in our data was $60 \mathrm{~Gy}$, which is higher than the recommended dose of PORT for lung cancer and may cause an excess radiation pneumonopathy or cardiac disease. Third, the proportion ratio of each component of C-SCLC may have prognostic significance and play an important role in the selection of therapies. However, the information of our pathologic diagnosis is not detailed enough to support the correspondent analysis.

\section{MATERIALS AND METHODS}

\section{Study population}

From Jan. 2004 to Dec. 2012, consecutive patients with pathologically diagnosed C-SCLC after complete surgery were enrolled. Patients with positive surgical margin were excluded. Initial evaluations included physical and hematological examination, chest CT scans or PET-CT, bronchoscopy, ultrasound, brain MRI and bone scan. Pathological diagnosis was based on specimens from surgery. C-SCLC was staged according to the 7th edition of the American Joint Committee on Cancer (AJCC) tumor-node-metastasis (TNM) classification system. The 
medical records and follow-up data of the patients were retrospectively analyzed.

\section{Surgery}

All patients underwent surgery of curative purpose. The types of surgical resection included wedge resection, sleeve resection, lobectomy and pneumonectomy.

\section{Radiotherapy}

The administration of radiation therapy was based on the attending radiation oncologist's decision and partially the surgeon's suggestion. The techniques of PORT included three-dimensional conformal radiotherapy (3D-CRT) and intensity modulated radiotherapy (IMRT). The clinical target volume (CTV) included the tumor bed, subcarinal nodes, ipsilateral mediastinum and ipsilateral hilum. The planning target volume (PTV) was defined as CTV plus 0.5 $\mathrm{cm}$ margins. PORT was administered with linear accelerator using 6 to $8 \mathrm{MV}$ X-rays at $1.8 \mathrm{~Gy}$-2.0Gy per fraction, 5 days per week, to a total prescription dose of $60 \mathrm{~Gy}$.

\section{Outcome measures}

Patients were followed-up every 3 months for the first year, then every 3 to 6 months thereafter. Overall survival (OS) was defined as the time between initial treatment and death or the last follow-up. Disease-free survival (DFS) was defined as the time between initial treatment and the relapse of disease or death or the last follow-up. Loco-regional recurrence-free survival (LRFS) was defined as the time between initial treatment and the recurrence of the primary tumor or regional lymph node, death or the last follow-up. Distant metastasis-free survival (DMFS) was measured from the date of initial treatment to the date of distant metastasis, death or the last follow-up.

\section{Statistical analysis}

The Kaplan-Meier method was used to estimate OS, DFS, LRFS and DMFS. The difference of survival between patients with different factors was compared using the log-rank test. Statistically significant difference was set as $p<0.05$.

\section{CONCLUSIONS}

For pathological $\mathrm{N}_{2}$ stage C-SCLC patients, PORT can significantly improve OS, DFS, LRFS and DMFS. Moreover, the patients with a large percent of metastatic lymph nodes also have significant beneficial survivals. In addition, the PORT group has higher OS and LRFS than the control when patients had a large number of DLN through surgery. Large-scale and multi-institution studies are needed to further evaluate the role of PORT in C-SCLC.

\section{CONFLICTS OF INTEREST}

Authors declare no conflicts of interests.

\section{FUNDING}

This study was supported by the Capital Health Development Research Grant for Youth Scholars (20114002-05), the Funding for Talents Training Project in Beijing (2012D009008000001), Beijing Hope Run Special Fund of Cancer Foundation of China (LC2016L03) and the PUMC Doctorial Innovation Fund (2015-1002-01-25).

\section{REFERENCES}

1. Nicholson SA, Beasley MB, Brambilla E, Hasleton PS, Colby TV, Sheppard MN, Falk R, Travis WD. Small cell lung carcinoma (SCLC): a clinicopathologic study of 100 cases with surgical specimens. Am J Surg Pathol. 2002; 26:1184-97.

2. Travis WD, Colby TV, Corrin B. Histological typing of lung and pleural tumors. 3rd ed. World Health Organization International Histological Classification of Tumors. Berlin: Springer; 1999.

3. Hage R, Elbers JR, Brutel de la Rivière A, van den Boscha JM. Surgery for combined type small cell lung carcinoma. Thorax. 1998; 53:450-3.

4. Fraire AE, Johnson EH, Yesner R, Zhang XB, Spjut HJ, Greenberg SD. Prognostic significance of histopathologic subtype and stage in small cell lung cancer. Hum Pathol. $1992 ; 23: 520-8$.

5. Mangum MD, Greco FA, Hainsworth JD, Hande KR, Johnson DH. Combined small-cell and non-small-cell lung cancer. J Clin Oncol. 1989; 7:607-12.

6. PORT Meta-analysis Trialists Group. Postoperative radiotherapy in non-small-cell lung cancer: systematic review and meta-analysis of individual patient data from nine randomised controlled trials. Lancet. 1998; 352:257-63.

7. Liu WS, Zhao LJ, Wang S, Gong LL, Liu ZY, Yuan ZY, Wang P. Benefits of postoperative radiotherapy in multimodality treatment of resected small-cell lung cancer with lymph node metastasis. Eur J Surg Oncol. 2014; 40:1156-62.

8. Burdett S, Stewart L. Postoperative radiotherapy in nonsmall-cell lung cancer: update of an individual patient data meta-analysis. Lung Cancer. 2005; 47:81-3.

9. Lally BE, Zelterman D, Colasanto JM, Haffty BG, Detterbeck FC, Wilson LD. Postoperative radiotherapy for stage II or III non-small-cell lung cancer using the surveillance, epidemiology, and end results database. J Clin Oncol. 2006; 24:2998-3006.

10. Dai H, Hui Z, Ji W, Liang J, Lu J, Ou G, Zhou Z, Feng Q, Xiao Z, Chen D, Zhang H, Yin W, He J, Wang L. Postoperative radiotherapy for resected pathological stage IIIA-N2 non-small cell lung cancer: a retrospective study 
of 221 cases from a single institution. Oncologist. 2011; 16:641-50.

11. Patel SH, Ma Y, Wernicke AG, Nori D, Chao KS, Parashar B. Evidence supporting contemporary postoperative radiation therapy (PORT) using linear accelerators in N2 lung cancer. Lung Cancer. 2014; 84:156-60.

12. Feld R, Rubinstein LV, Weisenberger TH. Sites of recurrence in resected stage I non-small-cell lung cancer: a guide for future studies. J Clin Oncol. 1984; 2:1352-8.

13. Thomas P, Rubinstein L. Cancer recurrence after resection: T1 N0 non-small cell lung cancer. Lung Cancer Study
Group. The Annals of Thoracic Surgery. 1990;49:242-6; discussion 6-7.

14. Pairolero PC, Williams DE, Bergstralh EJ, Piehler JM, Bernatz PE, Payne WS. Postsurgical stage I bronchogenic carcinoma: morbid implications of recurrent disease. The Annals of Thoracic Surgery. 1984; 38:331-8.

15. Urban D, Bar J, Solomon B, Ball D. Lymph node ratio may predict the benefit of postoperative radiotherapy in nonsmall-cell lung cancer. J Thorac Oncol. 2013; 8:940-6. 